\title{
Developing a new family of Newton-Secant method with memory based on a weight function
}

\begin{abstract}
In this paper, we construct an iterative method with memory based on the Newton-Secants method to solve nonlinear equations. This proposed method has fourth order convergence and costs only three functions evaluation per iteration and without any evaluation of the derivative function. Acceleration of the convergence speed is achieved by an appropriate variation of a free parameter in each step. This self-accelerator parameter is estimated using Newtons interpolation polynomial of third degree. The order of convergence is increased from 4 to 5.23 without any extra function evaluation. This method has the efficiency index equal to $5.2313 \approx 1.7358$. We describe the analysis of the proposed method along with numerical experiments including comparison with the existing methods. Finally, the attraction basins of the proposed method are shown and compared with other existing methods.
\end{abstract}

Keyword: Multi-point method; Nonlinear equations; Method with memory; R-order of convergence 